\title{
ELABORACIÓN Y OPTIMIZACIÓN DE UNA RED DE SENSORES ELECTROQUÍMICOS PARA UNA LENGUA ELECTRÓNICA ORIENTADA AL ANÁLISIS DE LECHE
}

\author{
Álvaro Á. Arrieta ${ }^{* a}$, Jorge A. Díaz ${ }^{b}$ Óscar C. Fuentes ${ }^{\mathrm{b}}$
}

\begin{abstract}
RESUMEN
En la actualidad, el uso de lenguas electrónicas ha cobrado importancia en la industria de alimentos, debido al crecimiento de la tecnología y su aprovechamiento en este sector. En el presente trabajo se muestra la elaboración y optimización de una red de sensores a base de polipirrol, el cual fue dopado con diferentes especies químicas para la modificación de una red de sensores de carbono como primera etapa de una lengua electrónica para el análisis de leche. Se analizó la estabilidad y variabilidad en la respuesta presentada por cada sensor mediante la aplicación de una técnica electroquímica conocida como voltametría cíclica, que se aplicó a través de dichos sensores a las muestras analizadas. Para el estudio de los datos se empleó un método estadístico de reconocimiento de patrones conocido como análisis de componentes principales (PCA) y para la optimización de los sensores modificados se usó el análisis de superficie de respuestas. Los resultados obtenidos demuestran que debido a la optimización de los sensores se generó una respuesta estable y con menor ruido en las señales respecto a los mismos sensores sin modificar. Se demostró la capacidad de esta red para responder diferente ante muestras de leche de diversa naturaleza.
\end{abstract}

Palabras clave: Lengua electrónica, sensores electroquímicos, polipirrol, leche.

\section{OPTIMIZATION OF THE SYNTHESIS OF POLYPYRROLE FOR THE ELABORATION OF A NETWORK OF SENSORS ELECTROCHEMICAL (ELECTRONIC TONGUE)}

\begin{abstract}
Nowadays, the use of electronic tongues has gained important in the food industry, due to the growth of the technology and its use in this sector. The present work shows the elaboration and optimization of an electrochemical sensors array based on polypyrrole doped with different chemical species for the modification of sensors as the first stage of an electronic tongue for the analysis of milk. The stability and variability was analyzed in the response presented by every sensor by means of the application of a technical electrochemistry known

${ }^{a}$ Departamento de Biología y Química, Universidad de Sucre, Carrera 28 \# 5-267 Barrio Puerta Roja, Sincelejo, Colombia, alvaroangel.arrieta@gmail.com.

${ }^{\mathrm{b}}$ Laboratorio DANM, Grupo de desarrollo y aplicación de nuevos materiales, Universidad Pontificia Bolivariana, Carrera 6 \#97A-99, Montería, Colombia.
\end{abstract}


as cyclic voltammetry that was applied across the above mentioned sensors to the analyzed samples. To study the data, patter recognition statistic method known as Principal Component Analysis (PCA) was used and for the optimization of the modified sensors was used response surface analysis. The results obtained shows that sensor optimization generate a response stable and with less noise on the same sensors without change. It was shown the capacity of this network to respond different according to milk samples.

Key words: Electronic tongue, electrochemical sensors, polypyrrole, milk.

\section{INTRODUCCIÓN}

La creciente demanda de productos en el sector alimenticio ha conllevado a que las empresas de esta industria se vean obligadas a buscar herramientas tecnológicas que permitan ofrecer mayor calidad en sus productos ${ }^{1}$, por lo que en la actualidad se han creado sistemas electrónicos bioinspirados como narices y lenguas electrónicas ${ }^{2-6}$. Gracias a estos dispositivos es posible discriminar diferentes tipos de bebidas ${ }^{7-11}$.

En general las lenguas electrónicas se encuentran conformadas por una red de sensores electroquímicos, que complementados con un equipo de análisis quimiométrico y herramientas de reconocimiento de patrones como el análisis de componentes principales, decodifica la información y luego clasifica las muestras.

La parte más importante de una lengua electrónica es la red de sensores, ya que es la encargada de extraer la información. Esta debe tener algunas características importantes, como selectividad cruzada, estabilidad y reproducibilidad en la señal dada, las cuales se consiguen implementando diferentes compuestos químicos a ciertas condiciones de elaboración.

En otros trabajos se han utilizado redes de sensores con sustratos constituidos por diferentes metales que han brindado resultados importantes en cuanto al reconocimiento de patrones frente a diferentes sustancias ${ }^{11-13}$. Además, se puede encontrar una serie de investigaciones en las que se usan electrodos de trabajo a base de polímeros conductores ${ }^{14-15}$, en las que se ha demostrado la excelente capacidad de estos materiales para ser utilizados como unidades sensibles. No obstante, la optimización del proceso de elaboración de los sensores, puede generar señales con características mejoradas en cuanto a la estabilidad y a la capacidad de clasificación de muestras particulares como la leche. Por lo tanto, en el presente trabajo se reporta la elaboración y optimización de una red de sensores modificados electroquímicamente con polipirrol y dopado con diferentes especies químicas, para ser aplicados en la discriminación y clasificación específica de muestras de leche. 


\section{PARTE EXPERIMENTAL}

Los reactivos empleados fueron adquiridos de Sigma-Aldrich: Cloruro de sodio, trifluorometanosulfonato de litio (TFMS), ferricianuro de potasio (FCP), ácido 10-canforsulfónico (ACS), hexacianoferrato de potasio (FCN), hexafluorofosfato de potasio (HFF), ftalocianina (FC), ácido trifluoroacético (TFA), ácido clorhídrico (ACH), pirrol (Py), perclorato de litio (PL), sulfato de litio (SL), dodecilbencenosulfonato de sodio (DBS), perclorato de tetrabutil amonio (TBAP), persulfato de amonio (PSA), ácido fosfotúngstico (PWA), ácido antraquinona 1,5-disulfónico hidrato de sal disódica (AQDD) y decanosulfonato de sodio (DSA). Las disoluciones se prepararon en agua ultra pura Milli-Q. El conjunto de muestras utilizadas en el desarrollo experimental estuvo constituido por leches de marcas comerciales (entera pasteurizada, entera, semidescremada deslactosada y semidescremada). El análisis se llevó a cabo a temperatura ambiente.

Los sensores usados como sustratos fueron adquiridos de DropSens; estos sensores están conformados por un electrodo de referencia de plata, un contraelectrodo de platino y un electrodo de trabajo de carbono (sustrato). La modificación de la superficie de los sensores comerciales se realizó a partir de pirrol y diferentes agentes dopantes en medio acuoso. La modificación se realizó por polimerización directa en el sustrato mediante cronoamperometría.

El proceso de optimización se realizó con diferentes dopantes y a diferentes condiciones experimentales: tiempos de polimerización de $(50,100$ y $300 \mathrm{~s})$, concentración de dopante de $(0,05 ; 0,10$ y $0,30 \mathrm{~mol} \mathrm{~L}-1)$ y concentración de pirrol de $(0,10 ; 0,20$ y $0,40 \mathrm{~mol} \mathrm{L-1})$. A los sensores se le aplicó 30 ciclos voltamétricos en una muestra de leche semidescremada para medir la pérdida de intensidad de la señal y medir su estabilidad, que luego alimentó el análisis de superficie de respuesta. Por otra parte, para obtener los valores de corriente, en el caso del análisis de componentes principales, se efectuaron 10 ciclos para estabilizar la respuesta del sensor antes de realizar cada análisis, el cual fue realizado siete veces (7 réplicas).

Las medidas electroquímicas se realizaron mediante el registro de voltamogramas con los sensores modificados sobre cada una de las muestras de leche. Estas medidas se obtuvieron aplicando un rango de potencial de $-1,0$ a $0,5 \mathrm{~V}$ y una velocidad de barrido de $0,1 \mathrm{~V} \mathrm{~s}^{-1}$.

Los análisis estadísticos realizados se basaron en análisis de superficie de respuesta y el análisis de componentes principales. Para el análisis de superficie de respuesta se usó el software Statgraphics Centurion XVI, en donde se estudió las condiciones de modificación de los sensores con pirrol mediante el uso de gráficas de superficies y la aplicación de un diseño factorial de $3^{3}$. Las gráficas de superficie de respuesta se construyeron con los valores del porcentaje de la caída de intensidad de las señales, lo que es evidencia de la estabilidad de las señales y con cada una de las variantes de los niveles de concentración y tiempo.

El análisis de componentes principales se realizó en Statistica 7,0, este análisis se llevó a cabo tomando como entrada los valores de corriente de las señales voltamétricas sin ningún tipo de pre-tratamiento. 


\section{RESULTADOS Y DISCUSIÓN}

\section{Estudios preliminares de la estabilidad de la señal en relación a las especies dopantes}

Antes de iniciar el proceso de optimización de la elaboración de los sensores, se estudiaron la respuesta de los sensores modificados con diferentes dopantes, para establecer una serie de dopantes que generen sensores estables. Los dopantes estudiados fueron: TFMS, FCP, ACS, FCN, HFF, FC, TFA, ACH, PL, SL, DBS, TBAP, PSA, PWA, AQQD y DSA. En esta etapa, donde aún no se tenían optimizadas las condiciones de elaboración, se utilizaron tiempos de polimerización de $50 \mathrm{~s}$, y concentración de pirrol y dopante de 0,1 y $0,5 \mathrm{~mol} \mathrm{~L}^{-1}$, respectivamente y cronoamperometría a $0,8 \mathrm{~V}$. Los resultados mostraron que la estabilidad depende en gran medida del agente dopante empleado. Adicionalmente, se obtuvieron diferentes valores de pérdida de intensidad para las señales voltamétricas extraídas por cada uno de los sensores modificados, mediante la aplicación de 30 ciclos voltamétricos frente a una solución de cloruro de sodio $0,1 \mathrm{~mol} \mathrm{~L}^{-1}$. En la figura 1 se presentan las respuestas de los sensores de polipirrol dopados con perclorato de litio y ácido 10-canforsulfónico. Se puede observar una amplia diferencia en la estabilidad de las señales, con valores de pérdida de estabilidad 1,20\% para PPy/PL y de 20,98\% para PPy/ACS.

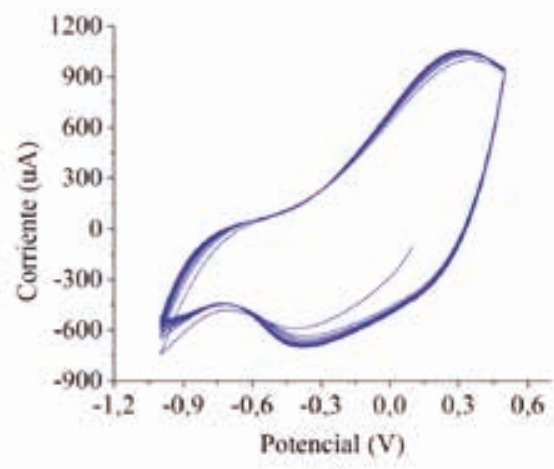

(a)

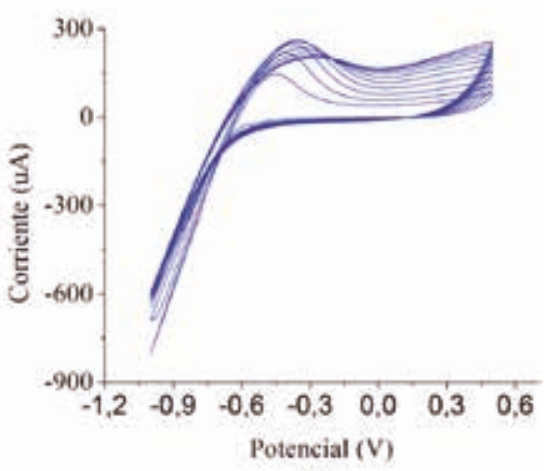

(b)

Figura 1. Respuestas voltamétricas frente a una solución de $\mathrm{NaCl}$ 0,1 mol L-1 con sensores: a) PPy/PL y b) PPy/ACS.

Este comportamiento puede ser asociado a las interacciones que pueden establecerse entre las especies dopantes y las cadenas de polipirrol que, en el caso de ser fuertes, pueden generar señales más estables debido a que no se da pérdida de dopante por atrapamiento electrostático (debido a las cargas) o físico (debido al tamaño de las especies).

Los dopantes con valores de pérdida de intensidad menores a 5,00\%, fueron considerados con buena estabilidad (PL, SL, DBS, TBAP, PSA, PWA, AQDD y DSA), los de mayor pérdida de intensidad fueron descartados (ACS, TFMS, FCP, FCN, HFF, FC, TFA, y ACH). 


\section{Evaluación de las respuestas voltamétricas frente a muestras de leche}

Una vez evaluado el comportamiento de los diferentes dopantes y su estabilidad, la red de sensores constituida por los dopantes con mayor estabilidad fueron expuestos a muestras de leche para estudiar su respuesta voltamétrica y establecer si se presentan procesos redox en sus señales que puedan dar información sobre las particularidades de las muestras debido a que estos procesos son el reflejo de las interacciones del material electroactivo (polipirrol) y el medio analizado en toda su complejidad química (muestra de leche).

En primera instancia se determinó la selectividad cruzada, que consiste en el hecho de que cada sensor responda de manera distinta frente a muestras con diferentes propiedades organolépticas y químicas, y que frente a una misma muestra de leche cada sensor muestre un comportamiento distinto, lo que evidencia que cada uno aporta información importante en la discriminación y clasificación de las muestras y que la red en conjunto puede ser capaz de diferenciar cada muestra. Para llevar a cabo este estudio se expuso la red de sensores a una muestra de leche pasteurizada. En la figura 2a, se presenta la respuesta obtenida y se observa que cada sensor registra una señal distinta, lo que nos indica que cada sensor está dando información sobre la muestra analizada.
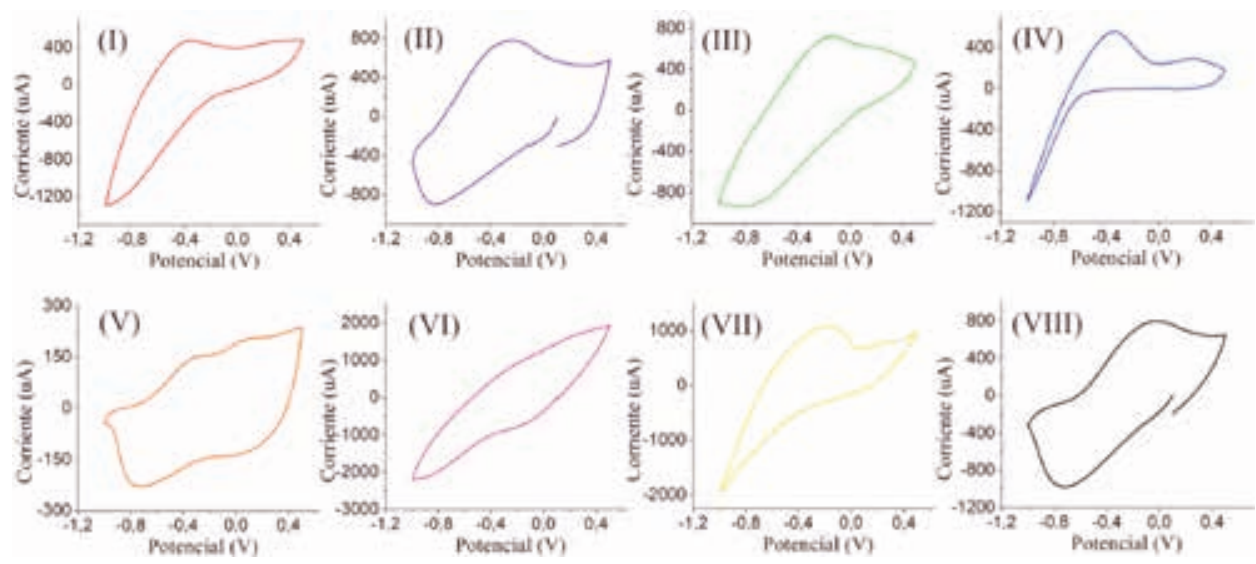

Figura 2. Señales voltamétricas extraídas de una muestra de leche pasteurizada: I) PPy/PL, II) PPy/AQDD, III) PPy/SL, III), IV) PPy/ DBS, V) PPy/ TBAP, VI) PPy/ PSA, VII) PPy/DSA y VIII) PPy/ PWA.

Por otra parte, se expuso un sensor modificado con polipirrol y perclorato de litio sobre diferentes muestra de leche. Se encontró que el sensor respondió de manera distinta frente a cada una de las muestras, lo que en definitiva permite a la red discriminar o clasificar muestras debido a su sensibilidad cruzada. Los perfiles voltamétricos extraídos por el sensor se ilustra en la figura 3. 


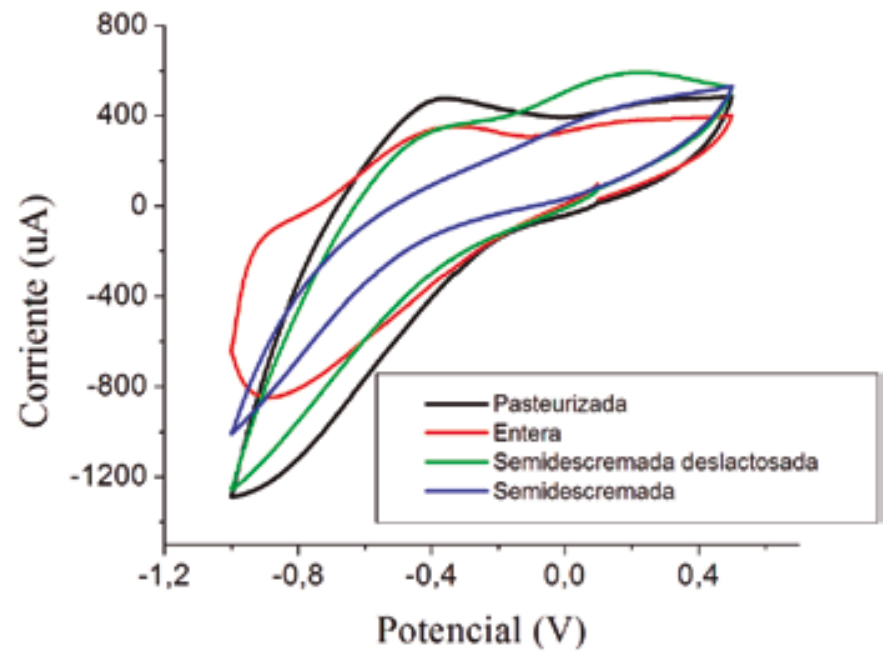

Figura 3. Voltamogramas registrados por un sensor a base de PPy/

PL sumergido en leche de diferente naturaleza.

En la tabla 1 se reporta los valores de los potenciales para los barridos anódicos y catódicos de los procesos óxido-reducción dispuestos en la figura 2. Cada uno de los potenciales demuestra la diversidad de información dada por la técnica de voltametría, además del uso de compuestos electroactivos como el pirrol y algunos iones dopantes.

Tabla 1. Potenciales debido a la capacidad electroactiva de la película PPy-Dopante Vs sustancia (leche).

\begin{tabular}{ccc}
\hline Voltamogramas & $\mathrm{E}_{1 / 2}$ Pico anódico $(\mathrm{V})$ & $\mathrm{E}_{1 / 2}$ Pico catódico $(\mathrm{V})$ \\
\hline I & $-0,42$ & ----- \\
II & $-0,22$ & $-0,82$ \\
III & $-0,17$ & $-0,77$ \\
IV & $-0,35$ & ----- \\
V & $-0,39$ y 0,18 & $-0,78$ y 0,20 \\
VI & ----- & $-0,09$ \\
VII & $-0,15$ & ----- \\
VIII & 0,09 & $-0,75$ \\
\hline
\end{tabular}

\section{Optimización del proceso de elaboración de la red de sensores}

Al comprobar la sensibilidad cruzada de la red de sensores, podemos inferir que ésta proporciona información que puede permitir la clasificación de muestras de leche. Sin embargo, para poder conseguir una mejor sensibilidad cruzada y una mejor estabilidad de los sensores, es necesario que el proceso de elaboración sea optimizado frente a lo que será su analito o muestras a analizar. 
En este sentido las gráficas de superficie de respuesta y las curvas de nivel permitieron analizar los efectos del tiempo de polimerización, concentración de pirrol y concentración del dopante sobre la estabilidad de las señales, la cual se utilizó como variable de salida. Lo anterior se realizó con la finalidad de obtener los valores óptimos de los niveles para cada uno de los factores utilizados en la elaboración de la red de sensores y descritos en la parte experimental.

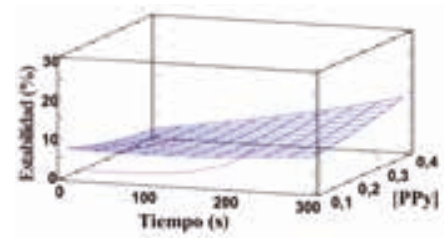

(a)

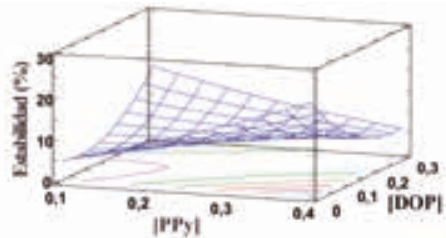

(c)
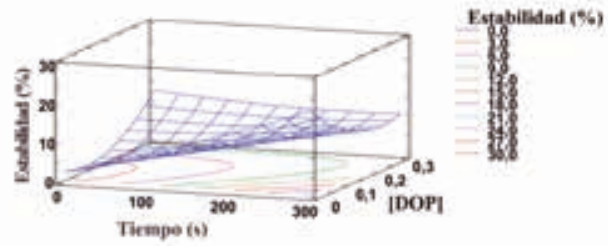

(b)

Figura 4. Superficies de respuesta y curvas de nivel para la estabilidad de las señales voltamétricas en función de la variación de los niveles de: a) tiempo Vs [PPy], b) tiempo Vs [Dop], c) [PPy] Vs [Dop] para las 27 combinaciones posibles.

En las gráficas de superficie de respuesta, que se muestran en la figura 4, donde la altura de las superficies representa los valores del porcentaje de estabilidad, que se determinaron por los porcentajes en la pérdida de la intensidad de las señales de los sensores; por tanto, valores más bajos de pérdida de intensidad representan mayor estabilidad. Este modelo de superficie de respuesta indica claramente el comportamiento de las variables en el proceso, demostrando así la proporcionalidad de los factores, es decir que al aumentar dos de los factores se puede afectar considerablemente la estabilidad de los sensores.

También, podemos observar que la concentración de dopante presenta un óptimo de estabilidad a valores intermedios próximos a $0,10 \mathrm{~mol} \mathrm{~L}^{-1}$. La concentración de pirrol para valores extremos presenta una tendencia a incrementar la inestabilidad de los sensores, y en este sentido la inestabilidad se ve aumentada por el incremento en el tiempo y contrarrestado por alta o baja concentración de dopante utilizado. En cuanto al tiempo, la tendencia exhibe mayor inestabilidad al exponer la celda a un tiempo de reacción prolongado. También, se puede observar que la zona más baja de la superficie de respuestas presentan los valores más adecuados para llevar a cabo la elaboración de los sensores, los cuales corresponden a tiempos de polimerización entre 100 y $150 \mathrm{~s}$, concentración de pirrol comprendida entre 0,18 y $0,20 \mathrm{~mol} \mathrm{~L}^{-1}$ y una concentración de dopante entre 0,10 y $0,15 \mathrm{~mol} \mathrm{~L}^{-1}$. Estos valores pueden confirmarse observando los mapas de contorno, donde la región cercana al valor óptimo es 
apreciable con el cambio en la curvatura de las líneas de contorno, lo que específicamente se presenta en la línea de color rosado de las curvas de nivel.

\section{Evaluación de la capacidad de discriminación de la red de sensores optimizada frente a muestras de leche comercial}

La capacidad de la lengua electrónica para discriminar y clasificar diferentes tipos de leche comercial fue determinada mediante el análisis de cuatro muestras de leche comercial. Una vez realizada las medidas, se hizo el tratamiento de datos mediante un análisis de componentes principales, la cual se puede observar en la figura 5.

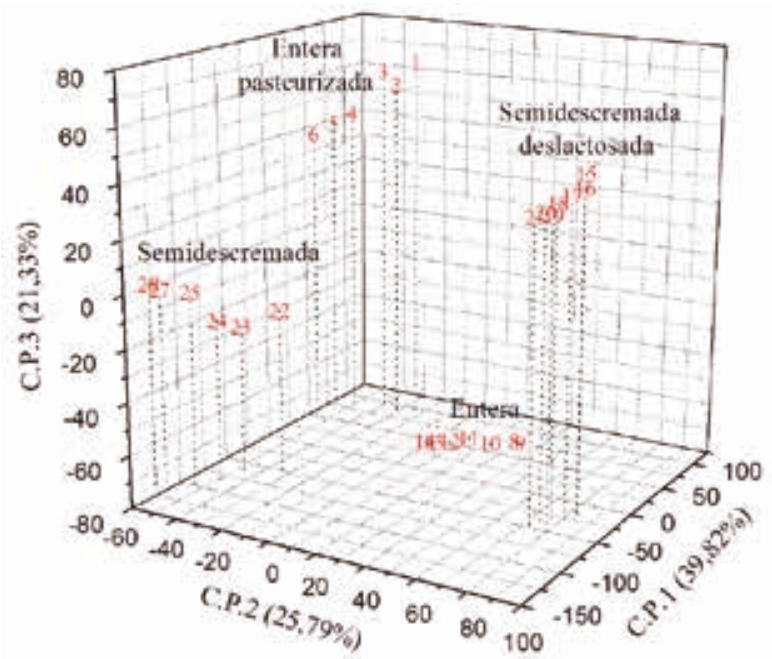

Figura 4. Representación de las tres primeras componentes principales de los valores obtenidos en muestras de diferentes tipos de leche.

Los resultados reportados en la figura 5, donde se muestran las tres primeras componentes principales, las que conjuntamente recogen un $86,84 \%$ de información presente en las muestras. La primera componente contiene la mayor cantidad de información con un $39,82 \%$, la segunda componente contiene el $25,79 \%$ y la tercera $21,33 \%$. Se puede apreciar claramente, una buena separación de las muestras en el plano representado, lo que indica una excelente capacidad de discriminación por la red de sensores elaborada y optimizada.

\section{CONCLUSIONES}

El uso de especies químicas electroactivas para la modificación de los sensores, permitió obtener señales estables debido a las interacciones dopante/PPy. Gracias a este comportamiento se incentiva la búsqueda de nuevos agentes modificadores con características que faciliten la obtención de repuestas electroquímicas variadas para la fabricación de lenguas electrónicas más eficientes. 
Mediante el análisis de superficie de respuesta se encontró una combinación óptima de los niveles en los valores experimentados, lo que permitió establecer para el proceso de modificación estudiado sensores que aportaran respuestas voltamétricas estables, empleando valores cercanos a la zona de un tiempo de $100 \mathrm{~s}$, concentración del monómero de 0,2 mol $\mathrm{L}^{-1} \mathrm{y}$ concentración de dopante de $0,10 \mathrm{~mol} \mathrm{~L}^{-1}$.

La técnica de voltametría cíclica implementada en este trabajo sirve correctamente como herramienta de recolección de información, dando así respuestas ricas y estables. Por lo que trae consigo una buena clasificación y reconocimiento exitoso de las muestras de leche.

\section{AGRADECIMIENTO}

Los autores agradecen el soporte financiero brindado por COLCIENCIAS (contrato $\mathrm{N}^{\circ}$. 0401-2013) y la Universidad Pontificia Bolivariana seccional Montería.

\section{REFERENCIAS BIBLIOGRÁFICAS}

1. Cappozzo JC, Koutchma T, Barnes G. Chemical characterization of milk after treatment with thermal (HTST and UHT) and nonthermal (turbulent flow ultraviolet) processing technologies. J Dairy Sci. 2015; 98(8):5068-79.

2. Parra V, Arrieta ÁA, Fernández-Escudero J-A, Rodríguez-Méndez ML, De Saja JA. Electronic tongue based on chemically modified electrodes and voltammetry for the detection of adulterations in wines. Sensors Actuat B-Chem. 2006;118(1-2):448-53.

3. García-Martínez T, Bellincontro A, de Lerma Mde L, Peinado RA, Mauricio JC, Mencarelli F, Moreno JJ. Discrimination of sweet wines partially fermented by two osmo-ethanol-tolerant yeasts by gas chromatographic analysis and electronic nose. Food Chem. 2011; 127(3):1391-6.

4. Simões da Costa AM, Delgadillo I, Rudnitskaya A. Detection of copper, lead, cadmium and iron in wine using electronic tongue sensor system. Talanta. 2014;129:63-71.

5. Marković K, Major N, Smola I, Levaj B, Krbavčić IP, Hruškar M, et al. Application of electronic tongue in isotonic sports drinks characterization and differentiation during storage. Croat J Food Sci Technol. 2011; 3 (2): 32-38.

6. Dias LA, Peres AM, Veloso ACA, Reis FS, Vilas-Boas M, Machado AASC. An electronic tongue taste evaluation: Identification of goat milk adulteration with bovine milk. Sensors Actuat B-Chem. 2009;136(1):209-17.

7. Moreno-Barón L, Cartas R, Merkoçi A, Alegret S, del Valle M, Leija L, et al. Application of the wavelet transform coupled with artificial neural networks for quantification purposes in a voltammetric electronic tongue. Sensors Actuat B-Chem. 2006;113(1):487-99.

8. Cetó X, González-Calabuig A, Capdevila J, Puig-Pujol A, del Valle M. Instrumental measurement of wine sensory descriptors using a voltammetric electronic tongue. Sensors Actuat B-Chem. 2015;207, Part B:1053-9. 
9. Cortina-Puig M, Muñoz-Berbel X, Alonso-Lomillo MA, Muñoz-Pascual FJ, del Valle M. EIS multianalyte sensing with an automated SIA system-An electronic tongue employing the impedimetric signal. Talanta. 2007; 72(2):774-9.

10. Campos I, Alcañiz M, Aguado D, Barat R, Ferrer J, Gil L, et al. A voltammetric electronic tongue as tool for water quality monitoring in wastewater treatment plants. Water Res. 2012;46(8):2605-14.

11. Escuder-Gilabert L, Peris M. Review: Highlights in recent applications of electronic tongues in food analysis. Anal Chim Acta. 14 de abril de 2010;665(1):15-25.

12. Novakowski W, Bertotti M, Paixão TRLC. Use of copper and gold electrodes as sensitive elements for fabrication of an electronic tongue: Discrimination of wines and whiskies. Microchem J. 2011;99(1):145-51.

13. Blanco CA, de la Fuente R, Caballero I, Rodríguez-Méndez ML. Beer discrimination using a portable electronic tongue based on screen-printed electrodes. J Food Eng. 2015;157:57-62.

14. Ha D, Sun Q, Su K, Wan H, Li H, Xu N, et al. Recent achievements in electronic tongue and bioelectronic tongue as taste sensors. Sensors Actuat B-Chem. 2015;207, Part B:1136-46.

15. Cetó X, Apetrei C, del Valle M, Rodríguez-Méndez ML. Evaluation of red wines antioxidant capacity by means of a voltammetric e-tongue with an optimized sensor array. Electrochim Acta. 2014;120:180-6. 